\title{
Pandemia 2020. Algunas consideraciones éticas
}

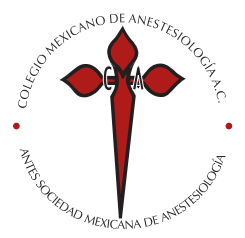

\section{Pandemic 2020. Some ethical considerations}

\author{
Dr. José Alejandro Esquivel-Guadarrama*
}

\begin{abstract}
RESUMEN. A lo largo de la historia de la humanidad, cada vez que se presenta una pandemia, se genera un clima de incertidumbre, debido al temor a lo impredecible de la situación, las posibilidades de afectación, el control que se pueda tener sobre ella y la manera en que esta pandemia afectará las condiciones socioeconómicas de un país. Una forma adecuada de respuesta por parte de las autoridades sanitarias ante situaciones como ésta, es estableciendo una política eficaz de comunicación, para permitir una corriente de confianza en la población. Los objetivos de esta comunicación antes o durante un brote epidémico son: educar, informar, recomendar, preparary prevenir. Ante una situación como la actual, nos enfrentamos a problemas que competen a la ética, y se crea un conflicto entre el bien individual y el bien común. Ante una pandemia, la eficacia necesaria para combatirla plantea la necesidad de priorizar a ciertos grupos, como los profesionales y trabajadores de la salud, quienes necesitarán estar más protegidos y atendidos, precisamente para que puedan responder a la demanda asistencial.
\end{abstract}

ABSTRACT. Throughout the history of humanity, each time a pandemic occurs, a climate of uncertainty is generated, due to fear of the unpredictability of the situation, the possibilities of affecting it, the control it may have over it, and the how this pandemic will affect the socioeconomic conditions of a country. An adequate form of response by health authorities in situations like this is establishing an effective communication policy to allow a flow of confidence in the population. The goals of this communication before or during an outbreak are: educate, inform, recommend, prepare, and prevent. Faced with a situation like the current one, we face ethical problems, and a conflict is created between the individual good and the common good. In the face of a pandemic, the effectiveness necessary to combat it raises the need to prioritize certain groups, such as health professionals and workers, who will need to be more protected and cared for, precisely so that they can respond to the demand for care.

\begin{abstract}
Palabras clave:
Pandemia, comunicación, bien individual, bien común, justicia, equidad, confianza.
\end{abstract}

Keywords:

Pandemic, communication, individual good, common good, justice, equity, trust.

* Comité Hospitalario de
Bioética, HMG Hospital
Coyoacán. Ciudad de México.

Solicitud de sobretiros:

Dr. José Alejandro

Esquivel Guadarrama.

HMG Hospital Coyoacán

Árbol del Fuego 80, Col. El Rosario, Coyoacán 04380, Ciudad de México. E-mail:

alejandro.esquivel@hmghospital.com

Recibido para publicación: 17-04-2020

Aceptado para publicación: 22-04-2020
C uando han ocurrido epidemias a lo largo de la historia de la humanidad, invariablemente se ha generado un clima de incertidumbre, comúnmente acompañado de pánico generalizado, sobre todo motivado por situaciones comunes a casi todas las pandemias: normalmente son impredecibles, porque no hay una cura o vacuna inmediata y porque no puede ser controlada en sus inicios por las instituciones de salud.

El resultado final puede ser la muerte de muchos seres humanos y cambios en la situación socioeconómica de bastantes sociedades. Ante la presencia de una epidemia, siempre se desarrollan incertidumbre y confusión social de manera paralela, las cuales deben ser atacadas, de manera inicial, con una estrategia adecuada de comunicación por parte de las instituciones de salud, tanto a nivel particular en cada país, como a nivel global, y así generar una corriente de confianza en la población.

Las estrategias de comunicación que desarrolle un país deben servir como parte integral de los planes nacionales contra una pandemia, para permitir que las ideas se transformen en acciones concretas que ayuden a aliviar los daños que de por sí ocasiona el padecimiento. Ante todas las posibles repercusiones de una epidemia, se deben realizar estrategias que incluyan planes detallados sobre qué se debe comunicar; cómo hacerlo; quién debe hacerlo y a quién se debe informar; además, se han de revisar en repetidas ocasiones para todas las fases de la pandemia y todo tipo de público.

Esta política de comunicación debe incluir lenguaje claro, directo, objetivos detallados, población a la que está destinada, mensajes clave y, sobre todo, planes de acción. Los objetivos de la comunicación antes o durante un brote son: educar, informar, recomendar, preparar y prevenir.

Se debe garantizar que la población, en parte a través de los medios de comunicación, reciba mensajes precisos relacionados con la salud, apoye las recomendaciones y que el personal sanitario involucrado esté informado, preparado y listo para actuar. 
Los medios de comunicación deben ser los espacios clave que contribuyan indirectamente a reducir la cantidad de contagios y evitar el pánico, pero si no se encuentran bien coordinados con las autoridades sanitarias responsables (locales, regionales y nacionales), pueden ocasionar retrasos en el control de la epidemia, al afectar y contribuir a disminuir la confianza de la gente.

La alianza para mantener informadas a las comunidades entre gobierno, medios de comunicación y organismos mundiales, debe integrar al ciudadano digital, por lo que las redes sociales en nuestra época (Facebook, Twitter, Instagram, entre otros) sirven para difundir la información y como plataforma de discusión de todos los temas relacionados con la pandemia.

Las epidemias son un problema de salud pública y de comunicación en la sociedad actual, y como tal deben entenderse. En la era digital, el riesgo es que la interacción entre la población por medio de las redes sociales sin una adecuada estrategia de comunicación por parte de las autoridades de salud, intensifique la emergencia hasta convertirla en pánico social. Por lo que es muy importante una adecuada intervención de gobiernos, organizaciones de la sociedad civil y medios de comunicación, para movilizar digitalmente a la población en beneficio de los objetivos de interés general.

La población espera y demanda recibir información precisa y adecuada. El usuario de Internet se enfrenta a una sobrecarga de información; por lo que se requiere de una presencia sólida por parte de las autoridades de salud, que les permita posicionar las fuentes oficiales y destacar entre los miles de contenidos que filtran datos imprecisos, rumores y teorías equivocadas sobre el agente causal y sus repercusiones, los cuales generan confusión y desconfianza en la población.

Existen cinco principios para la planificación de la comunicación en brotes epidémicos propuestos por la Organización Mundial de la Salud:

- Confianza: se debe establecer un lazo de confianza entre la población y quienes manejan la política de comunicación.

- Anuncio temprano: el primer anuncio oficial relacionado con la pandemia debe llegar en tiempo real, de manera simple y con el mayor alcance posible.

- Transparencia: la información debe ser clara, de fácil comprensión y completa.

- Planificación: en el contexto de una pandemia, debe haber una planificación previa de lo que se pretende comunicar.

- Tomar en consideración al público receptor: entender lo que la gente piensa y desea es esencial, las convicciones y creencias de la población deben ser tomadas en cuenta.

El manejo de los datos también implica aspectos éticos: la información epidemiológica detallada puede ser usada con fines políticos, diversos a su intención original, lo que la desvirtuaría y, por otro lado, la información limitada conduce al desconocimiento de los profesionales y ciudadanos sobre los riesgos a los que pueden estar enfrentándose.

Cada sociedad cuenta con una moral, que es el conjunto de valores que definen lo que la gente cree que está bien y lo que cree que está mal. Esta moralidad se refleja en las conductas que se aceptan y las que se rechazan en esa sociedad.

En la realidad, es frecuente que aparezcan conflictos que hay que resolver, esto es cuando confrontamos dos hechos, dos procesos que están bien, los dos de acuerdo con nuestras reglas morales. Ambos aspectos son buenos en sí mismos, pero entran en conflicto, ya que debemos resolver este problema y optar por uno de ellos. Estos dilemas son los que resuelve la ética, que analizará los valores y circunstancias que hay detrás de cada uno de ellos, sus posibles beneficios y riesgos asociados para poder tomar una decisión.

En este momento en el mundo, estamos viviendo una situación excepcional, un estado de alarma generalizado, propiciado por la epidemia por el coronavirus SARS-CoV-2, responsable del COVID-19, y por toda la información que alrededor del tema se presenta.

Ante una situación como la actual, nos enfrentamos a problemas que competen a la ética, ya que comprometen valores que, de una u otra manera, entran en conflicto cuando se asumen conductos personales y se plantean acciones que buscan soluciones generales. En otras palabras, se crea un conflicto entre el bien individual y el bien común.

En las respuestas personales, frente a una epidemia o riesgo de pandemia, entran en juego los derechos individuales a la libertad, a la salud y aun el derecho a la vida. Sin embargo, los derechos individuales se enfrentan a las necesidades de los demás miembros de la sociedad en la que vivimos.

La experiencia de la crisis actual, nos permite mencionar algunas situaciones/ conflictos en los que es necesaria una mirada desde la ética:

- El conflicto entre los derechos individuales frente a la protección de la salud colectiva: por ejemplo, el aislamiento domiciliario de casos y contactos, la interrupción de actividades que no se consideren prioritarias o el cierre de comercios, colegios y espacios públicos son posibles decisiones en las que se genera un desafío entre distintos niveles de derechos.

- En situaciones de pandemia ante un virus desconocido, se genera la incertidumbre del tratamiento médico y se puede caer en la tentación de iniciar manejos que aún no han sido debidamente probados, al iniciarse protocolos de investigación que no reúnen todos los requisitos que desde el punto de vista ético se requieren, a veces presionados por la urgencia de la situación, obviamente faltando a principios como el de la no maleficencia. 
— Las vacunas ante un virus desconocido tardan en producirse, y se crea la posibilidad de que los contratos para su adquisición se hagan cuando aún no hay suficiente conocimiento sobre la posible evolución de la pandemia y sobre la seguridad de esas vacunas. Las autoridades de salud de los diferentes países tienen que soportar una gran presión, puesto que todos los integrantes de la sociedad intentan influir para ser incluidos en los grupos susceptibles de ser vacunados. Es frecuente que de manera inicial la capacidad de producción de las vacunas normalmente sea limitada, pero también existe la posibilidad de que haya países que vean reducida su capacidad de adquirirlas, porque su situación económica se ha visto afectada por todos los gastos inherentes a una pandemia.

— La necesidad de priorizar el tipo de pacientes que reciban tratamientos especializados limitados en su disponibilidad, como lo estamos viendo en la actualidad en diversas partes del mundo y probablemente en el futuro cercano en nuestro país. (Por ejemplo, camas en Unidades de Cuidados Intensivos (UCI) o ventiladores).

- Clásicamente, existen criterios internacionalmente aceptados de ingreso en Unidades de Cuidados Intensivos, (no se detallarán en este momento, porque no es el tema principal del presente documento).

Pero estos criterios cobran relevancia a raíz de la publicación hace unos días de la Guía Bioética de Asignación de Recursos de Medicina Crítica, documento que desde su publicación y hasta el momento de escribir este artículo, han generado polémica por los conceptos que vierten en su contenido, y que han ocasionado dentro de las reacciones a su publicación múltiples comentarios e, incluso, ya se ha retirado la versión electrónica del portal del Consejo de Salubridad General y se intenta suplir con un «Proyecto de Guía de Triaje para la Asignación de Recursos en Medicina Crítica».

El documento en mención, intentó ser una guía bioética para la toma de decisiones en el triage, cuando la demanda de recursos de medicina crítica rebase a la capacidad real de otorgarlos, situación que se ha presentado ya en otros países; como por ejemplo en Italia y que en un futuro cercano, desgraciadamente muy cercano, se presentará en nuestro país.

Esta guía básicamente se dividía en dos partes. En la primera, se trataba de dar sustento bioético a la toma de decisiones al asignar recursos de medicina crítica en el momento en que la capacidad real fuera rebasada; mientras que en la segunda parte se trataba de describir el procedimiento que debía seguirse para la asignación de dichos recursos.

En dicha guía, que no es diferente a las existentes en España o en Italia, ante una situación de falta de recursos, se establece privilegiar los ingresos en la UCI de pacientes con prioridad 1 , en aquellos hospitales que dispongan de dispositivos de cuidados intermedios, dejando estos últimos para los pacientes con prioridad 2. Los pacientes de prioridades 3 y 4, en casos de crisis, no ingresarían en Unidades de Cuidados Intensivos.

En caso de complicaciones, o una mala evolución tanto clínica como funcional, se plantea el retiro terapéutico pronto en pacientes no recuperables, para no caer en futilidad e iniciar en ellos la aplicación de medidas paliativas. Para iniciar la reasignación de los recursos previamente retirados en otro paciente, usando criterios pronósticos, en donde se habla, entre otras cosas, de cantidad de «vidas por completarse».

En ese mismo escenario y en el desarrollo de las diversas fases de la pandemia, la decisión sobre qué momento y a qué paciente se le van a interrumpir los cuidados especializados, siguiendo criterios de disponibilidad de recursos, edad, pronóstico, expectativas y calidad de vida, todo ello dentro de un contexto de discusión de valores éticos, debe ser consensuada con los diversos sectores de la sociedad involucrados, y en donde el papel de las comisiones de bioética locales y nacionales deben tener un papel rector determinante.

Existen documentos a nivel internacional que ya plantean estas disyuntivas, por ejemplo, ante dos pacientes similares, se debe priorizar a la persona con más años de vida ajustados a la calidad (AVAC) o QALY (Quality-Adjusted Life Year). Son un indicador combinado del estado de la salud que aúna cantidad y calidad de vida. Priorizar la mayor esperanza de vida con calidad.

Estamos en espera de que este documento se revise y se emita en el corto plazo la versión debidamente modificada y consensuada con las instancias involucradas en la toma de decisiones y en la asignación de recursos cuando empiecen a escasear.

Lo que sí es un imperativo ético es que las decisiones de adecuación terapéutica que finalmente decidan tomarse, de manera ideal, deben consensuarse con el paciente cuando sea posible y/o sus familiares.

En casos extremos, como la pandemia a la que nos enfrentamos, se debe también considerar como un valor el deber de obediencia a las normas de salud pública que establezcan las autoridades sanitarias del país, ya que las garantías de todos los afectados dependen de su cumplimiento.

En las decisiones públicas que tomen las autoridades sanitarias para enfrentar la gravedad de una epidemia y el riesgo de una pandemia, se comprometen valores que, de acuerdo a como se jerarquicen, determinarán la conveniencia de estas decisiones. Debemos siempre partir de que el valor principal en esta situación es, evidentemente, la protección de la salud de la población, mediante medidas eficaces basadas en las mejores pruebas científicas existentes. El principio bioético de justicia es el fundamento primordial en las decisiones de salud pública.

La justicia debe incluir, por supuesto, la distribución de los recursos existentes con criterios de equidad, asignándolos de acuerdo con las necesidades y urgencias independientemente de los recursos económicos de los distintos grupos o partes de una sociedad a la que están destinados. 
Justicia y equidad se unen a la solidaridad, ya no como una actitud personal, sino como una obligación social que lleva a favorecer la atención de los más vulnerables de una sociedad. Sin embargo, ante una pandemia, la eficacia necesaria para combatirla plantea la necesidad de priorizar a ciertos grupos, como los profesionales y trabajadores de la salud, quienes necesitarán estar más protegidos y atendidos, precisamente para que puedan responder a la demanda asistencial.

La actitud de los médicos, enfermeras y de todos los profesionales y trabajadores de la salud, hace necesario considerar otros valores que también entran en conflicto ante las epidemias. Un profesional es, en primer lugar, un ciudadano y como tal tiene los mismos derechos y deberes que todos. Los profesionales de la salud se encuentran ante disyuntivas: una responsabilidad específica que se fundamenta en un contrato social implícito, en la reciprocidad por el privilegio de haber recibido su educación médica, y en la obligación moral estipulada en el juramento tácito hecho al concluir su preparación académica. Lo anterior se traduce en un conjunto de características que conocemos como las virtudes del profesional de la salud, entre las cuales están la postergación personal, el deber de no abandono y el «deber de tratar».

Éstos son valores que pueden entrar en conflicto con otros valores y deberes como son las responsabilidades familiares y de otros proyectos personales. Lo expresado para los médicos atañe, evidentemente en su dimensión propia, a todos los profesionales de la salud, técnicos y otros trabajadores.

Mientras que, de manera individual, los profesionales sanitarios también se enfrentan a dilemas éticos personales, que no siempre son analizados por la sociedad en su conjunto: el miedo al contagio propio o de familiares cercanos, o la necesidad de cuidar a miembros enfermos de su familia les pondrá en el dilema de acudir a su puesto de trabajo o permanecer en casa. Esto se puede aplicar a otros colectivos profesionales como el de la policía, los bomberos o los conductores de transportes públicos.

El colapso de los servicios de salud, el rápido y exponencial crecimiento de contagios está poniendo en evidencia la fragilidad del sistema de salud del país. Además, la escasez de insumos necesarios como ventiladores mecánicos, de mascarillas e indumentaria clave para combatir el COVID-19 ha hecho que empiecen a generarse conflictos, cada vez más frecuentes conforme la pandemia evolucione en los próximos días.

Todo lo anterior está generando que la labor de los profesionales de la salud involucrados se vuelva cada vez más difícil, debiendo tomar decisiones arriesgadas bajo altos niveles de estrés y durante largas jornadas de trabajo.

Contemplar desde antes de que se presenten las crisis, los aspectos éticos y hacer bien explícitas las razones que motivan a la toma de decisiones, que en su momento pueden generar inconformidad y polémica, contribuirá a evitar que durante la fase de respuesta se puedan producir daños a determinados grupos de la población, pérdida de confianza o descoordinación entre los miembros de la sociedad afectados.

Como todas las crisis, una situación de pandemia es una buena oportunidad para demostrar nuestros mejores principios: justicia, solidaridad, equidad, transparencia, $\mathrm{y}$ reciprocidad, tanto dentro de nuestro propio país como a nivel global, y esto tendrá que verse reflejado a corto plazo, una vez que pase la pandemia y que pueda ser analizada con detenimiento toda la serie de decisiones y acciones tomadas en estos momentos.

\section{LECTURAS RECOMENDADAS}

1. Huníades UM, Dalmacia NB, Levy MJ, et al. Comunicación efectiva y ética en casos de epidemias y pandemias. Arch Venez Puer Ped. 2016;79:113-117.

2. Beca JP. Problemas éticos en situaciones de catástrofes. Marzo de 2010. Publicación del Centro de Bioética de la Facultad de Medicina de la Universidad del Desarrollo, Chile. Disponible en: https://medicina.udd. cl/centro-bioetica/files/2010/10/parral.pdf.

3. Organización Panamericana de la Salud. Programa Regional de Bioética, Departamento de Sistemas y Servicios de Salud. Orientación ética sobre cuestiones planteadas por la pandemia del nuevo coronavirus (COVID-19). 20 Mar 2020.

4. Informe del Ministerio de Sanidad sobre los aspectos éticos en situaciones de pandemia: el SARS-CoV-2. Madrid, España.

5. Pellegrino ED. Moral choice, the good of the patient, and the patient's good. In: Moskop JC, Kopelman L. Ethics and critical care medicine. Philosophy and medicine. Vol. 19. Dordrecht: Springer; 1985.

6. WHO Guidelines on ethical issues in public health surveillance. Organización Mundial de la Salud 2017. Pautas de la OMS sobre la ética en la vigilancia de la salud pública. Organización Panamericana de la Salud 2017.

7. Bickenbach J. Disability and health care rationing. In: Zalta EN (ed.). The Stanford encyclopedia of philosophy. Metaphysics Research Lab, Stanford University; 2016.
8. Recomendaciones de la Red de América Latina y el Caribe de Comités Nacionales de Bioética Ante las investigaciones biomédicas por la pandemia de enfermedad infecciosa por coronavirus COVID-19.

9. Gherardi CR. La muerte intervenida: una visión comprensiva desde la acción sobre el soporte vital. Disponible en: http://www.aabioetica.org/ reflexiones/axa263.ht

10. Giordano A. Ley de voluntades anticipadas: implicancias en la práctica de la Medicina Intensiva. En: Tejera D, Taranto E, Soto J, Manzanares W. Bioética en el paciente grave. Montevideo, Uruguay: Editorial Cuadrado; 2017.

11. White DB, Katz MH, Luce JM, Lo B. Who should receive life support during a public health emergency? Using ethical principles to improve allocation decisions. Ann Intern Med. 2009;150:132-138.

12. Arias B P. La ética durante las crisis sanitarias: a propósito de la pandemia por el virus h1n1. Rev Esp Salud Pública. 2009;83:489-491.

13. Vergano M, Bertolini G, Giannini A, et al. Raccomandazioni di etica clinica per l'ammissione a trattamenti intensivi e per la loro sospensione, in condizioni eccezionali di squilibrio tra necessità e risorse disponibili. Società Italiana di Anestesia Analgesia Rianimazione e Terapia Intensiva. Disponible en: http://www.siaarti. it/SiteAssets/News/COVID19 
14. Grupo de Trabajo de Bioética de la SEMICYUC. Recomendaciones éticas para la toma de decisiones en la situación excepcional de crisis por pandemia COVID-19 en las Unidades de Cuidados Intensivos. Sociedad Española de Medicina Intensiva, Crítica y Unidades Coronarias. Disponible en: https://semicyuc.org/wp-content/ uploads/2020/03/.pdf

15. Beca JP, Salas SP. Ethical and health issues posed by the recent Ebola epidemic: what should we learn? Rev Med Chil. 2016;144:371-376.

16. White DB, Katz MH, Luce JM, Lo B. Who should receive life support during a public health emergency? Using ethical principles to improve allocation decisions. Ann Intern Med. 2009;150:132-138.

17. Guía Bioética de Asignación de Recursos de Medicina Crítica. Consejo de Salubridad General, México, 2020.

18. Beca JP. Una mirada ética a las epidemias. Revista Academia Número 9, Facultad de Medicina CAS-UDD.
19. Randall JC, Kross EK, Stapleton RD, The importance of addressing advance care planning and decisions about do-not-resuscitate orders during novel coronavirus 2019 (COVID-19). JAMA. 2020 March 27. doi: 10.1001/jama.2020.4894.

20. Murthy S, Leligdowicz A, Adhikari NK. Intensive care unit capacity in low-income countries: a systematic review. PLoS One. 2015;10:e0116949. doi: 10.1371/journal.pone.0116949.

21. American Thoracic Society Bioethics Task Force. Fair allocation of intensive care unit resources. American Thoracic Society. Am J Respir Crit Care Med. 1997;156:1282-1301.

22. Broome J. Selecting people randomly. Ethics. 1984;95:38-55.

23. Lin Y, Zhu M, Su Z. The pursuit of balance: An overview of covariateadaptive randomization techniques in clinical trials. Contemp Clin Trials. 2015;45:21-25.

24. La bioética ante la pandemia del COVID-19. Pronunciamiento. Manuel H. Ruiz de Chávez, Comisionado Nacional de Bioética. México, 12 marzo 2020. 